\title{
Hypertension Before Pregnancy and Infant Outcomes: Secondary Analysis of North Carolina Pregnancy Risks Assessment Monitoring System (PRAMS) Data
}

Forgive Avorgbedor ( $\square$ avorgbe@live.unc.edu )

University of North Carolina at Chapel Hill https://orcid.org/0000-0002-5028-7105

\section{Susan Silva}

Duke University School of Nursing

James A Blumenthal

Duke University School of Medicine

\section{Seonae Yeo}

University of North Carolina at Chapel Hill

\section{Elizabeth Merwin}

Duke University School of Nursing

\section{Diane Holditch-Davis}

Duke University School of Nursing

\section{Research article}

Keywords: chronic hypertension, preterm infants, black women maternal age, small for gestational age infants

Posted Date: August 10th, 2020

DOI: https://doi.org/10.21203/rs.3.rs-50601/v1

License: (a) (i) This work is licensed under a Creative Commons Attribution 4.0 International License. Read Full License 


\section{Abstract}

Background: Chronic hypertension complicates birth outcomes. This secondary analysis of data from the North Carolina 2009-2011 Pregnancy Risks Assessment Monitoring System (PRAMS) examined factors associated with infant outcomes (preterm birth and small for gestational age) among women with hypertension before pregnancy to determine if the maternal age and race/ethnicity moderated the effect of preexisting hypertension on preterm birth and small for gestational age infants.

Methods: We performed logistic regression to determine whether hypertension before pregnancy (HTN, $n=292$; non-HTN controls, $n=2625$ ), maternal age and race/ethnicity (Black vs Non-Black) and their interactions with hypertension before pregnancy predicted preterm birth and small for gestational age infants compared to normotensive women.

Results: Results indicated that women with hypertension before pregnancy had significantly higher rates of preterm birth ( $29.8 \%$ vs. $21.3 \%)$ and small for gestational infants $(23.0 \%$ vs. $17.9 \%)$ compared to nonHTN controls without adjusting for covariates. HTN had an effect on preterm birth that was independent of other maternal risk factors $(a O R=1.31)$ after adjusting for covariates . Being Black was associated with a greater likelihood of preterm birth $(a O R=1.55)$.

Conclusion: Hypertension before pregnancy is more likely to be associated Black race and with adverse birth outcomes. Black women are especially likely to experience poor birth outcomes. Continuous education to encourage all women and especially women of color and women with chronic illness to access preconception care is needed

\section{Background}

Chronic hypertension (HTN) (i.e., blood pressure [BP] above 140/90 Hg prior to pregnancy or before 20 weeks gestation) is one of the leading causes of maternal mortality and infant morbidity worldwide (1-3). About $8 \%$ of women of reproductive age are affected with HTN and this rate is increasing due to delayed childbearing $(4,5)$.

The adverse impact of HTN on fetal outcomes is consequential and likely to increase even more due to a higher incidence of older women giving birth $(1,4,6)$. Greater maternal age is associated with HTN which increases adverse birth outcomes including preterm birth and small for gestational age (SGA) infants (710).

In addition to advanced maternal age, Black race/ethnicity birth outcomes are worse than White women $(11,12)$. Although the poor birth outcomes of Black women may be affected by many other factors including low socioeconomic status, Black women are more likely to have HTN (13). The extent to which older age and Black race/ethnicity may contribute to adverse birth outcomes in women with HTN prior to conception is not known. Therefore, we sought to determine whether maternal factors moderate the relationship between HTN and infant outcomes in a large and diverse sample of pregnant women with 
and without HTN before conception obtained from the 2009-2011 Pregnancy Risk Assessment Monitoring Surveillance (PRAMS) dataset from North Carolina.

\section{Methods}

The PRAMS dataset compiled state-specific, population-based data to monitor behaviors, conditions and experiences before and during pregnancy, and shortly after childbirth among women who delivered liveborn infants $(14,15)$. PRAMS surveys consisted of core questions, optional questions, and state-specific questions. The PRAMS data included women who delivered a live infant in a given year. About 1700 new mothers from North Carolina were sampled every year for PRAMS. The PRAMS staff mailed the first questionnaire to women 2-6 months after they delivered a live infant. The mailed questionnaires were followed-up by telephone calls. The survey data was then linked to the state's birth certificate data and weighted for sample design, non-response, and non-coverage $(14,15)$. North Carolina sampled more infants with low birth weight to allow the data to be representative of the general population, because most infants have normal birth weight. More details on PRAMS are available at www.cdc.gov/prams.

\section{Analysis Sample}

The analysis sample was comprised of 2917 women with singleton births and their infants born in North Carolina. Women who did not have data available to determine their blood pressure status were excluded. HTN status was determined by self-report; 292 women reported a history of HTN, and 2625 women indicated that their blood pressure was normal and served as non-HTN controls. This secondary analysis was approved by Duke University Health System Institutional Review Board.

\section{Measures}

Hypertension Status: The North Carolina questionnaire to assess HTN before pregnancy was based on response to a single item: "I visited a health care worker to be checked or treated for high blood pressure" at any time during the 12 months before being pregnant.

(Yes, No). Yes, was classified as having HTN during the 12 months prior to pregnancy, while the no HTN control group was comprised of women who reported no history of HTN prior to pregnancy.

Infant Outcomes: Preterm birth and SGA infants were obtained from hospital records. Preterm birth was defined as birth before 37 weeks gestation and SGA was defined by an estimated infant birth weight less than 10th percentile for gestational age. Candidate moderating risk factors were maternal age (in years) and race/ethnicity ("1" = Black; "0" = Non-Black [includes white, Hispanics and others]). Moderator analyses were conducted to identify subgroups of women with HTN before pregnancy whose infants are at greater risk for preterm birth and SGA. 


\section{Data Analysis}

Non-directional statistical tests were performed with the a priori significance set at $p<0.05$ for each test. Data analyses were conducted using SAS 9.4 software (Cary, North Carolina). Descriptive statistics were used to summarize the maternal and infant characteristics and two infant outcomes for each HTN (HTN versus non-HTN control).

Bivariate Analyses. HTN differences in sample characteristics and infant outcomes were tested using bivariate chi-square tests for categorical characteristics and General Linear Models (GLMs, due to unequal sample sizes per group) for scalar characteristics. Chi-square tests were also used to test for group differences on the two infant outcomes, without moderators and/or covariates included in the analysis.

Moderators and Covariates. Candidate moderators were maternal age and race/ethnicity. Thus, the initial multivariate model included these maternal characteristics and their interactions with the HTN status. Covariates were other maternal characteristics for which a significant difference was detected between the HTN and non-HTN control in the above bivariate between-group analyses of the sample characteristics. Only covariate main effects were included in the initial multivariate models.

Multivariate Analyses. Logistic regression models were used to determine whether HTN/non-HTN control, maternal age and race/ethnicity and their interactions with HTN predicted each infant outcome, after covarying for other maternal characteristics. The initial regression included HTN status, candidatemoderators and their interaction with HTN status, and potential maternal covariates. The initial regression model was then reduced to a final model using an iterative backward selection process to eliminate non-significant covariates, interactions, and/or candidate-moderators $(p>0.05)$. If a significant interaction was detected, the components of the interaction were included in the final model regardless of statistical significance. HTN was always retained in the final model regardless of statistical significance since this was an independent variable of interest. Adjusted odds ratios (aOR) and their $95 \%$ confidence intervals $(\mathrm{Cl})$ were used to address effect size and clinical relevance in the final model.

\section{Statistical Power}

Power calculations indicated that a sample size of 2917 (HTN: N=292; non-HTN control $N=2625$ ) provided at least $80 \%$ power to test for the influence of HTN along with two candidate-moderators (age and race/ethnicity) and their interactions with HTN on each infant outcome using logistic regression models with the two-tailed level of significance set at $<0.05$. This determination was based on the assumption of medium effect sizes $(a O R=2.47)$ with seven or fewer moderator and/or covariate terms in each final model. Power calculations did not take into account multiple tests and multiple outcomes.

\section{Results}


Table 1 provides descriptive statistics for maternal and infant characteristics. Women with HTN compared to non-HTN controls were more likely to be Black (48.6\% vs. $20.3 \%$ ). Compared to normotensive controls, the women with HTN had a significantly higher rate of preterm birth $(29.8 \%$ vs. $21.3 \%)$, gave birth with more infants with SGA $(23.0 \%$ vs. $17.9 \%)$, and lower overall birth weight ( $45.6 \%$ vs. 29.2\%) (North Carolina PRAMS data oversampled women who had low birth weight to assure data represent the entire population). Women with HTN also had infants with significantly lower mean birth weights and gestational ages compared to non-HTN control women.

Table 1. Characteristics of Women with and without Chronic Hypertension (Controls) and their Infants

\begin{tabular}{|c|c|c|c|c|c|}
\hline \multirow{3}{*}{$\begin{array}{l}\text { Maternal and Infant Characteristics } \\
\text { Age, in years, mean } \forall S D\end{array}$} & \multirow{2}{*}{\multicolumn{2}{|c|}{$\begin{array}{l}\text { HTN } \\
N=292\end{array}$}} & \multirow{2}{*}{\multicolumn{2}{|c|}{$\begin{array}{l}\text { Controls } \\
N=2625\end{array}$}} & \multirow{3}{*}{$\begin{array}{l}p \\
0.68\end{array}$} \\
\hline & & & & & \\
\hline & 27.8 & $\otimes 7.0$ & 27.7 & $\otimes 6.0$ & \\
\hline Black race/ethnicity, $n(\%)$ & 142 & $(48.6)$ & 533 & $(20.3)$ & $<.001$ \\
\hline 12 or less years of education, $n(\%)$ & 155 & $(53.4)$ & 1086 & $(41.4)$ & $<.001$ \\
\hline Married, $n(\%)$ & 136 & $(46.6)$ & 1633 & $(62.2)$ & $<.001$ \\
\hline Infection kidney/bladder, $n(\%)$ & 73 & $(25.1)$ & 576 & $(22.0)$ & 0.22 \\
\hline Caesarean, $n(\%)$ & 112 & $(38.4)$ & 884 & $(33.7)$ & 0.11 \\
\hline NICU admission, $n(\%)$ & 72 & $(25.2)$ & 538 & $(20.8)$ & 0.08 \\
\hline Hospital stay > 2 days, $n(\%)$ & 156 & $(54.7)$ & 1055 & $(40.8)$ & $<.001$ \\
\hline Female gender, $n(\%)$ & 141 & $(48.3)$ & 1340 & $(51.1)$ & 0.37 \\
\hline Birth weight, g, mean $\otimes S D$ & 2727.1 & $\llbracket 853.8$ & 3005.7 & $₫ 819.7$ & $<.001$ \\
\hline Gestational age, wks. mean $\mathbb{S} D$ & 36.8 & $凶 3.8$ & 37.6 & $\otimes 3.4$ & $<.001$ \\
\hline Preterm birth, $n$ (\%) & 87 & $(29.8)$ & 558 & $(21.3)$ & $<.001$ \\
\hline Small for gestational age, $n(\%)$ & 67 & $(23.0)$ & 469 & $(17.9)$ & 0.04 \\
\hline *Low birth weight, $g, n$ (\%) & 133 & $(45.6)$ & 767 & $(29.2)$ & $<.001$ \\
\hline
\end{tabular}

HTN=Hypertension; Low birth weight=Less than 2500 grams; SD=Standard deviation; Chi-square tests for categorical variables and General Linear Models for scalar characteristics. * Low birth weight was oversampled in the NC PRAMS data.

Maternal characteristics did not significantly moderate the influence of HTN on infant outcomes (Table 2). HTN had an effect on preterm birth that was independent of other maternal risk factors $(a O R=1.31)$ after adjusting for covariates (see Table). Being Black was associated with a greater likelihood of preterm 
birth ( $a O R=1.55)$. HTN was not associated with SGA infants (see Table 2) after adjusting for covariates and neither maternal race/ethnicity and age were moderators of SGA.

In summary, HTN is associated with preterm births, but not SGA after adjustment for covariates. Being Black associated with HTN-but no other maternal characteristics associated with HTN.

\section{Table 2. Final Logistic Regression Results for Infant Outcomes}

\begin{tabular}{|lllll|}
\hline Outcome & Explanatory Variable & AOR & AOR 95\% CI & $p$ \\
\hline \multirow{2}{*}{ Preterm birth } & HTN (Control ref) & 1.31 & $1.00,1.73$ & $<0.05$ \\
& Black race/ethnicity (Non-Black ref) & 1.55 & $1.15,1.91$ & $<.0001$ \\
& Married (Unmarried ref) & 0.70 & $0.58,0.85$ & 0.0002 \\
\hline \multirow{2}{*}{ SGA } & HTN (Control ref) & 1.32 & $0.98,1.76$ & 0.07 \\
& Maternal age (older to younger, ref) & 0.98 & $0.97,1.00$ & 0.06 \\
& Married (Unmarried ref) & 0.78 & $0.63,0.96$ & 0.02 \\
\hline
\end{tabular}

AOR=adjusted odds ratio; $95 \% \mathrm{Cl}=95 \%$ Confidence Interval; SGA=small for gestational age, ref =reference; Non-Black=White, Hispanic and others.

\section{Discussion}

In this secondary analysis, we found that HTN before pregnancy was associated with adverse outcomes, preterm birth and being Black is associated with HTN. However, there was no interaction between HTN before pregnancy, maternal age, or race/ethnicity.

The risk of preterm birth was almost 5 times higher in women with HTN and about $50 \%$ of women with HTN were at higher risk of having SGA infants (16). Similarly, in the present study, women with HTN had higher rates of preterm births, but not SGA when adjusted for covariates. In our study, the rates of preterm birth and SGA infants were higher in women with HTN when compared to women without HTN. However, the effect of HTN on SGA infants was minimized when other variables were added to the model, indicating that among women with HTN, other maternal characteristics may increase adverse birth outcomes. This finding is consistent with previous studies, some studies reported association between HTN and SGA $(16,17)$, and others found increased rates of preterm birth and SGA infants in women with $\operatorname{HTN}(7,18)$.

Black women were at increased risk of having preterm birth infants regardless of their HTN status. Black women are at increased risk for adverse birth outcomes regardless of their HTN status while the risk for adverse birth outcomes increased in white women but only if they had HTN. These results confirm 
findings of previous studies $(2,19-21)$, which also found that Black race is a precursor to adverse birth outcomes including preterm birth.

While our study had a number of strengths, including a large sample including a number of women who had HTN before pregnancy and the sample included in this study is often excluded from other studies due to preexisting HTN. The study has several important limitations including missing data on HTN status for about $47 \%$ of sample of women who had singleton births and reliance on self-reported HTN status based on their reporting that they received treatment for their HTN before pregnancy.

\section{Conclusion}

HTN before pregnancy is more likely to be associated Black race and with adverse birth outcomes. Black women are especially likely to experience poor birth outcomes. Continuous education to encourage all women and especially women of color and women with chronic illness to access preconception care is needed.

\section{Abbreviations}

aOR, Adjusted Odds Ratio; BP, Blood Pressure; HTN, Chronic Hypertension; Cl, Confidence Intervals; GLMs, General Linear Models; SGA, Small for Gestational Age; PRAMS, Pregnancy Risk Assessment Monitoring Surveillance

\section{Declarations}

\section{Ethics approval and consent to participate}

Ethical approval was obtained from the Duke University Health System Institutional Review Board. Pregnancy Risk Assessment Monitoring Surveillance data are publicly available thus informed consent was waived because it is a secondary data.

\section{Consent for publication}

Not applicable

\section{Availability of data and materials}

Pregnancy Risk Assessment Monitoring Surveillance data are available through the Centers for Disease Control and Prevention and the state of North Carolina.

\section{Competing interests}

All authors report no conflicts of interest

\section{Funding}


No direct funding for this research project

\section{Authors' contributions}

FA, SS, JAB, YS, EM, DHD actively participated in the study design, data analysis and drafting and proofreading of this manuscript.

\section{Acknowledgements}

We acknowledge the North Carolina Department of Health Statistics for providing the data for the study and the Duke University School of Nursing, which supported this work. This work was completed, in part, to meet the dissertation requirements for the $\mathrm{PhD}$ of the first author. In addition, this work was partially supported by grants HL122836, HL130237-02 and HL130237-02S1 from the National Heart, Lung and Blood Institute. We also want to acknowledge T32NR007091: Interventions for Preventing \& Managing Chronic Illness, which funds the postdoctoral position of the first author. We thank Mr. Richard Sloane and Dr. Patrick Smith for statistical consultation and Dr. Lynne Lewallen for her editorial assistance.

\section{References}

1. Bramham K, Parnell B, Nelson-Piercy C, Seed PT, Poston L, Chappell LC. Chronic hypertension and pregnancy outcomes: systematic review and meta-analysis. BMJ (Clinical research ed). 2014;348:g2301.

2. Chappell LC, Enye S, Seed P, Briley AL, Poston L, Shennan AH. Adverse perinatal outcomes and risk factors for preeclampsia in women with chronic hypertension: a prospective study. Hypertension. 2008;51(4):1002-9.

3. Ono Y, Takagi K, Seki H, Takai Y, Samejima K, Matsunaga S, et al. Neonatal outcome in infants of chronically hypertensive mothers. The journal of obstetrics and gynaecology research. 2013;39(6):1142-6.

4. Bateman BT, Bansil P, Hernandez-Diaz S, Mhyre JM, Callaghan WM, Kuklina EV. Prevalence, trends, and outcomes of chronic hypertension: a nationwide sample of delivery admissions. American journal of obstetrics and gynecology. 2012;206(2):134 e1-8.

5. Seely EW, Ecker J. Chronic Hypertension in Pregnancy. Circulation. 2014;129(11):1254-

6. Ankumah NA, Cantu J, Jauk V, Biggio J, Hauth J, Andrews W, et al. Risk of adverse pregnancy outcomes in women with mild chronic hypertension before 20 weeks of gestation. Obstetrics and gynecology. 2014;123(5):966-72.

7. Broekhuijsen K, Ravelli AC, Langenveld J, van Pampus MG, van den Berg PP, Mol BW, et al. Maternal and neonatal outcomes of pregnancy in women with chronic hypertension: a retrospective analysis of a national register. Acta obstetricia et gynecologica Scandinavica. 2015;94(12):1337-45.

8. Kenny LC, Lavender T, McNamee R, O'Neill SM, Mills T, Khashan AS. Advanced maternal age and adverse pregnancy outcome: evidence from a large contemporary cohort. PloS one. 
2013;8(2):e56583.

9. Luke B, Brown MB. Elevated risks of pregnancy complications and adverse outcomes with increasing maternal age. Human reproduction (Oxford, England). 2007;22(5):1264-72.

10. Madi JM, Araujo BF, Zatti H, Rombaldi RL, Madi SR, de Zorzi P, et al. Chronic hypertension and pregnancy at a tertiary-care and university hospital. Hypertension in pregnancy : official journal of the International Society for the Study of Hypertension in Pregnancy. 2012;31(3):350-6.

11. Bryant AS, Worjoloh A, Caughey AB, Washington AE. Racial/ethnic disparities in obstetric outcomes and care: prevalence and determinants. American journal of obstetrics and gynecology. 2010;202(4):335-43.

12. Byrd DR, Katcher ML, Peppard P, Durkin M, Remington PL. Infant mortality: explaining black/white disparities in Wisconsin. Maternal and child health journal. 2007;11(4):319-26.

13. Bryant AS, Seely EW, Cohen A, Lieberman E. Patterns of pregnancy-related hypertension in black and white women. Hypertension in pregnancy : official journal of the International Society for the Study of Hypertension in Pregnancy. 2005;24(3):281-90.

14. Shulman HB, D'Angelo DV, Harrison L, Smith RA, Warner L. The Pregnancy Risk Assessment Monitoring System (PRAMS): Overview of Design and Methodology. American journal of public health. 2018;108(10):1305-13.

15. Shulman HB, Gilbert BC, Lansky A. The Pregnancy Risk Assessment Monitoring System (PRAMS): current methods and evaluation of 2001 response rates. Public Health Reports. 2006;121(1):74.

16. Catov JM, Nohr EA, Olsen J, Ness RB. Chronic hypertension related to risk for preterm and term small for gestational age births. Obstetrics and gynecology. 2008;112(2 Pt 1):290-6.

17. Haelterman E, Breart G, Paris-Llado J, Dramaix M, Tchobroutsky C. Effect of uncomplicated chronic hypertension on the risk of small-for-gestational age birth. American journal of epidemiology. 1997;145(8):689-95.

18. Sibai BM. Chronic hypertension in pregnancy. Obstetrics and gynecology. 2002;100(2):369-77.

19. Dole N, Savitz DA, Siega-Riz AM, Hertz-Picciotto I, McMahon MJ, Buekens P. Psychosocial factors and preterm birth among African American and White women in central North Carolina. American Journal of Public Health. 2004;94(8):1358-65.

20. Goldenberg RL, Culhane JF, lams JD, Romero R. Epidemiology and causes of preterm birth. The lancet. 2008;371(9606):75-84.

21. Vintzileos AM, Ananth CV, Smulian JC, Scorza WE, Knuppel RA. The impact of prenatal care in the United States on preterm births in the presence and absence of antenatal high-risk conditions. American journal of obstetrics and gynecology. 2002;187(5):1254-7. 